\title{
Healthcare utilization for antinuclear antibody and rheumatoid factor labs in the assessment of musculoskeletal pain in children and adolescents - A claims analysis and chart review
}

\author{
Patricia Rosillo, Debra Canter, Eyal Muscal, Marietta De Guzman, Jeannine Yoon and Angelo Giardino* \\ Texas Children's Hospital, Academic General Pediatrics, Baylor College of Medicine, TX, USA
}

\section{Background}

According to the National Bureau of Economics Research, Canada spends far less of its gross domestic product on health care (10. $4 \%$ versus $16 \%$ in the U. S. ) yet performs better than the U.S. on infant mortality rate and life expectancy [1]. Lowering healthcare utilization and costs are important goals for primary care and subspecialty providers. In March 2012, the Agency for Healthcare Research and Quality (AHRQ) published a meta-analysis of the utility of lab testing in musculoskeletal pain evaluation and screening of rheumatic diseases in children and adolescents ${ }^{2}$. The review analyzed 28 pediatric articles and concluded that current evidence does not support the use of antinuclear antibody (ANA) and rheumatoid factor (RF) testing as a screening for rheumatic diseases in the primary pediatric care setting. Despite this and other articles with similar recommendations, it seems that inappropriate use of these labs continues to be a persistent problem inpediatrics [2-4]. Referring pediatric patients to rheumatology for positive labs with a normal exam and complaints of intermittent joint pain generates multiple unnecessary referrals that prolong wait times for new patients with active pathology. Additionally, new patients are seen with severe joint damage and contractures that are not referred earlier as their "arthritis labs are negative." This practice may waste a significant amount of money annually and contributes to higher healthcare utilization [5].

In order to quantify this problem, we choose to review the healthcare utilization and costs for children and adolescents with juvenile idiopathic arthritis (JIA) in the Texas Children's Hospital (TCH) Health Plan during January 1 to December 31, 2011. JIA is the most common rheumatic disease of childhood and it is also one of the more frequent chronic illnesses in childhood that cause short and long term disability [6,8]. JIA is defined in children that are younger than 16 years old and have persistent joint pain and swelling for at least 6 weeks [6]. A positive ANA has long been associated with several inflammatory rheumatic diseases [5,7]. Although it is included among classification criteria for diseases like systemic lupus erythromatosus and other inflammatory connective tissue diseases, it is not specific to these conditions [5,7]. A positive ANA in absence of arthritis, malar rash, or cytopenias is of no clinical significance [5,7]. In patients with JIA a positive ANA can be found in about $18-77 \%$ of patients $[1,6]$. However, after a patient has been diagnosed by a pediatric rheumatologist with JIA, this test is used to predict patients' risk for uveitis and determines the interval for their ophthalmologic evaluations $[1,6]$.
JIA shares many clinical features and ICD-9 code with adult rheumatoid arthritis. They even share the same ICD-9 code. In adults, a positive test for RF is $97 \%$ specific and has a predictive value for $80 \%$ [7]. Yet JIA pathophysiology, presentation, and lab assessment is not identical to its adult counterpart. In children, a positive RF is found in only about $5-10 \%$ of children clinically diagnosed with polyarticular disease $[1,3,6]$. Taking this into consideration, a primary care provider should refer a child or adolescent for evaluation if he or she meets the above criteria and infection and malignancy have been excluded $[5,6]$. Lab studies are only confirmatory in JIA and have no place in screening schema. We hypothesized that lab utilization practices seen in outpatient rheumatology clinic would correlate with significant annual cost for an individual health plan.

\section{Methods}

We reviewed administrative claims data from January 1 to December 31, 2011 for TCH Health Plan members' ages 0-17 years old to identify unique members with the arthritis-related ICD-9 codes (Table 1) and CPT codes for ANA (86038 and 86039) and RF (86431). The TCH Health Plan is a health maintenance organization (HMO) that serves exclusively children with Medicaid or the Children's Health Insurance Program (CHIP). This was done to describe the use of these

Table 1. Arthritis-related codes.

\begin{tabular}{|l|l|}
\hline ICD-9 code & Description \\
\hline 696.xx & Psoriatic arthropathy \\
\hline 713.xx & Arthropathy \\
\hline 714.xx & $\begin{array}{l}\text { Inflammatory arthropathy, juvenile idiopathic arthritis, rheumatoid } \\
\text { arthritis }\end{array}$ \\
\hline 715.xx & Osteoarthrosis \\
\hline 716.xx & Kaschin-Beck disease, osteoarthropathy \\
\hline 720.xx & Unspecified inflammatory spondylopathy, ankylosing spondylopathy \\
\hline 737.xx & Synovitis and tenosynovitis \\
\hline 726.xx & Rheumatism unspecified \\
\hline
\end{tabular}

Correspondence to: Angelo P. Giardino, MD, PhD, SVP/Chief Quality Officer, Texas Children's Hospital, Professor \& Section Head, Academic General Pediatrics, Baylor College of Medicine, Houston, TX, USA, Tel: 832-824-1128, E-mail: apgiardi@texaschildrens.org

Received: October 04, 2016; Accepted: October 31, 2016; Published: November 03,2016 
labs across the TCH Health Plan. Data capture for unique members with diagnosis code 714. $\mathrm{xx}$ (JIA diagnosis code) was collected to include other health service data, such as service provider specialty, radiology and laboratory services, and cost for ANA and RF. We set out to identify coding patterns for primary care providers when using the ICD-9 code 714. $\mathrm{xx}$ for the first and second diagnoses. Available charts for members with JIA diagnosis code were reviewed by a pediatric rheumatologist to assess for correct use of the code and appropriateness of referral. This project was approved by the Baylor College of Medicine IRB.

\section{Results}

Claims analyses for 1,574 members with rheumatologic diagnosis codes were identified and reviewed. A multitude of patients had multiple autoimmune lab tests performed and some members had labs repeated up to five times (i. e. a member with intermittent knee swelling and pain that resulted in a diagnoses of torn a meniscus after seeing multiple providers including primary care, ER, and orthopedic providers) (Table 2). Fifty nine percent of the ANA and $76 \%$ of the RF test were ordered by primary care physicians.

Taking in consideration the total annual costs for the insurance plan laboratory evaluation, this gives an average cost of $\$ 313$ for each ANA claim and $\$ 98$ for each RF claim (Table 3).

Further analyses identified 174 unique members with diagnosis code for JIA (714. xx). These members had 81 claims for ANA and 54 claims for RF. Chart review of these 174 JIA members showed that approximately $47 \%(n=83)$ of them were already established patients at $\mathrm{TCH}$ and $58 \%(\mathrm{n}=91)$ had a newly coded diagnosis of JIA. Only $42 \%$ $(n=38)$ of the newly coded members were referred to rheumatology sub-specialist for evaluation. Of the newly coded members $30 \%(n=27)$ were not referred. Of these groups, ANA and RF tests were ordered for $n=28$ patients but did not lead to a subsequent referral to a TCH pediatric rheumatologist (Figure 1). The reminding $42 \%(n=38)$ of the newly coded JIA group where referred to a pediatric rheumatology subspecialist a diagnosis of JIA was confirmed in $47 \%$ of them (Figure 2). Of these referred children, $10 \%(n=4)$ initially had a negative ANA and their referrals were delayed (range 6-18 months). This delay may have contributed to the progression of severe joint deformity and disability documented in their medical record upon presentation to rheumatology sub-specialist.

Table 2. Utilization rates of ANA and RF testing among pediatric health plan members Jan 1- Dec 312011 by associated diagnosis code.

\begin{tabular}{|c|c|c|c|c|}
\hline \multirow{3}{*}{\begin{tabular}{|l} 
Associated Dx Code \\
Non Arthritis \\
\end{tabular}} & \multicolumn{4}{|c|}{ Test } \\
\hline & \multicolumn{2}{|c|}{$\begin{array}{c}\text { ANA claims } \% \text { of test } \\
\text { ordered }\end{array}$} & \multicolumn{2}{|c|}{$\begin{array}{c}\text { RF claims } \% \text { of tes } \\
\text { ordered }\end{array}$} \\
\hline & 1,202 & $89 \%$ & 672 & $85 \%$ \\
\hline Arthritis & 155 & $11 \%$ & 116 & $15 \%$ \\
\hline 714.xx & 67 & $43 \%$ & 47 & $41 \%$ \\
\hline Other arthritis & 88 & $57 \%$ & 69 & $59 \%$ \\
\hline TOTALS & 1357 & $100 \%$ & 788 & $100 \%$ \\
\hline
\end{tabular}

Table 3. Total claims and costs for ANA and RF testing among Pediatric Health Plan Members Jan 1-Dec 312011 by associated diagnosis code.

\begin{tabular}{|c|c|c|c|c|c|c|}
\hline Unique Members & \multicolumn{3}{|c|}{ ANA } & \multicolumn{3}{|c|}{ RF } \\
\hline Associated Dx Code & $\mathrm{n}$ & $\%$ & Cost & $\mathrm{n}$ & $\%$ & Cost \\
\hline Non Arthritis & 1,316 & $87 \%$ & $\$ 381,731.90$ & 700 & $84 \%$ & $\$ 64,779.89$ \\
\hline Arthritis & 191 & $13 \%$ & $\$ 90,318.11$ & 129 & $16 \%$ & $\$ 16,056.19$ \\
\hline 714.xx M & 81 & $42 \%$ & $\$ 37,562.00$ & 54 & $42 \%$ & $\$ 6,383.03$ \\
\hline Other arthritis & 110 & $58 \%$ & $\$ 52,756.11$ & 75 & $58 \%$ & $\$ 9,673.16$ \\
\hline TOTALS & 1507 & $100 \%$ & $\$ 472,050.01$ & 829 & $100 \%$ & $\$ 80,836.08$ \\
\hline
\end{tabular}

\section{Newly-coded JIA}

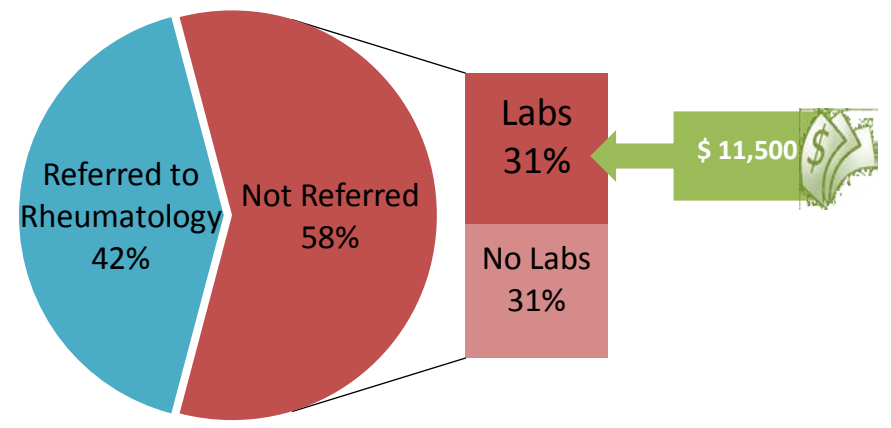

Figure 1. Claim analysis revealed the majority of patients (58\%) with a new ICD-9 code 174.xx for JIA were not referred to a pediatric rheumatologist (RHE) (N=91).

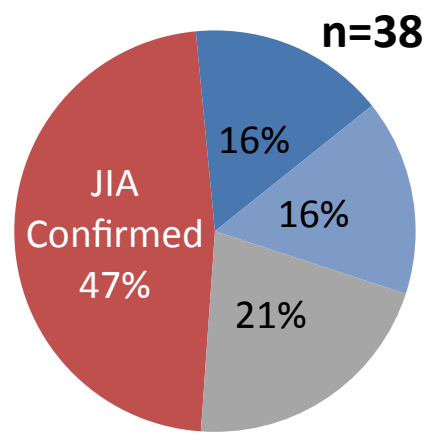

MSK pain

Figure 2. JIA diagnosis was confirmed in less than half of coded cases referred $(n=38)$.

\section{Discussion}

We describe a preliminary pilot study. That assesses the frequency and expenditures associated with ANA and RF testing among members of an insured pediatric population. We supplemented claims date with clinical chart review of children with JIA ICD-9 code to assess appropriate use and potential impact of lab studies on a timely rheumatology referral and clinical course. Although there is currently insufficient evidence to support the use of RF and/or ANA as screening or diagnostic tests in children with suspected JIA, we found that these tests continue to be utilized for this purpose by pediatric primary care providers. Analyzing of the administrative claims and charts, documented that the code for JIA was often inappropriately assigned to order X-rays and magnetic resonance imaging (i. e. patient with brain cancer or an HIV patient with complains of bone or joint pain). Fifty nine percent of the ANA and $76 \%$ of the RF test were ordered by primary care physicians. As hypothesized, ANA/RF testing was frequently and repetitively inappropriately utilized by primary care providers. The cost of unnecessary ANA claims is difficult to assess as ANA is also used for evaluation of other autoimmune conditions, beyond the scope for this study. However, a pediatric primary care provider should never use a $\mathrm{RF}$ to screen for arthritis in children and adolescents. This could save about $\$ 61,435.42(76 \%$ of $\$ 80,836.08)$ a year to the TCH health plan (small urban health plan). Furthermore, the inappropriate use of these labs of screening for rheumatologic conditions contributed to observe delays in referrals (range 6-18 months) of four patients that presented with severe joint disease, disability, and deformity.

There are limitations to our data collection and conclusions. 
Retrospective reviews of claims databases pose a series of methodological challenges, some of which are unique to this data source. Issues include clinical relevance, reliability of validity of data and data linkages. Also, chart review was not done in a systematic way and some important data was not recorded effectively. Other limitations included sample selection, and variable definitions. We limited to Table 1 codes for ANA and RF and did not take into account other bundled or reflex tests, which may have overlooked subjects for inclusion, or affected the valuation of associated costs. Our study chart review were restricted to members with diagnosis code JIA, 714. $\mathrm{xx}$ and not all members getting lab testing; this resulted in the identification of potential sampling bias and perhaps incomplete understanding of lab and referral patterns. Finally, given the high volume of patients and associated claims received each year, we limited the scope of inquiry to a "snap-shot" of claims data during a 1-year period. Although looking at health care cost data from one year is thought to be sufficient to identify major sources for health care expenditures, more stable estimates would be obtained by combining health care claims data for 24 or more months. We believe cost and inappropriate utilization patterns are probably even underestimated by our design limitations. Our findings emphasize the need for continuing educational efforts to disseminate guidelines and recommendations for diagnostic testing in children with suspected JIA and other musculoskeletal complaints. Not only for the unnecessary cost to our health care, but also to prevent morbidity to children and adolescents that have delayed referrals for having negative ANA/RF test.

\section{Future directions}

As our preliminary evaluation revealed apparent overuse and misuse of ANA and RF testing, further work is planned to address methodological issues. Specifically, we will examine claims for a period of more than a year, include CPT codes associated with bundled test, and will extend our chart review to primary and secondary diagnosis codes for other JIA-related conditions. We will evaluate the predictive value of ANA and RF testing for rheumatologic complaints, as well as the impact on delay in care and increased costs associated with testing by examining test results. We will complete an extended claims analysis and chart review that will involve revisiting study variable definitions, and development of a standardized data collection tool. We have submitted an IRB amendment to permit this expanded evaluation, and are refining our methodology so to develop a project timeline to enable robust re-analysis and dissemination of results.

We feel these refinements will enhance the validity of our analysis and interpretation. We then may be able to identify target populations of providers for which reimbursement modules, clinical guidelines, and subsequent educational activities can be developed to reduce healthcare costs and ultimately improve quality of care and patient outcomes.

\section{References}

1. Comparing the U.S. and Canadian Health Care Systems. [www.nber.org/bah/fall07/ w13429.html]

2. Wong KO, Bond K, Homik J, Ellsworth JE, Karkhaneh M, et al. (2012) Antinuclea antibody, rheumatoid factor, and cyclic-citrullinated peptide tests for evaluating musculoskeletal complaints in children. AHRQ Comparative Effectiveness Reviews 50: 1-31. [Crossref]

3. Smith JA (2009) Testing for Rheumatological Diagnoses in Children. Eur Paediatr Rev 3: 30-34. [Crossref]

4. Correll CK, Spector LG, Zhang L, Brinstadt BA, Vehe RK (2014) A137: Appropriateness of rheumatology lab studies and referrals among general pediatricians. Arthritis \& Rheumatology 66: S180.

5. Breda L, Nozzi M, De Sanctis S, Chiarelli F (2010) Laboratory testing in the diagnosi and follow-up of pediatric rheumatic diseases: an update. Semin Arthritis Rheum 40 53-72. [Crossref]

6. Cassidy J, Petty RE (2011) Textbook of pediatric rheumatology (6 $6^{\text {th }}$ edn). Chronic arthritis in childhood 13: 211-235.

7. Meyer O (2003) Evaluating inflammatory joint disease: how and when can autoantibodies help? Joint Bone Spine 70: 433-447. [Crossref]

8. Sacks JJ, Helmick CG, Luo Y, Ilowite NT, Bowyer S (2007) Prevalence of and annua ambulatory health care visits for pediatric arthritis and other rheumatologic conditions in the United States in 2001-2004. Arthritis Rheum 57: 1439-1445. [Crossref]

9. Helmick CG, Felson DT, Lawrence RC, Gabriel S, Hirsch R, et al. (2008) Estimates of prevalence of arthritis and other rheumatic conditions in the United States. Arthritis Rheum 58: 15-25. [Crossref]

10. Harrold LR, Salman C, Shoor S, Curtis JR, Asgari MM, et al. (2013) Incidence and prevalence of juvenile idiopathic arthritis among children in a managed care population, 1996-2009. J Rheumatol 40: 1218-1225. [Crossref]

11. van Rossum M, van Soesbergen R, de Kort S, ten Cate R, Zwinderman AH, et al. (2003) Anti-cyclic citrullinated peptide (anti-CCP) antibodies in children with juvenile idiopathic arthritis. $J$ Rheumatol 30: 825-828. [Crossref]

Copyright: (C2016 Rosillo P. This is an open-access article distributed under the terms of the Creative Commons Attribution License, which permits unrestricted use, distribution, and reproduction in any medium, provided the original author and source are credited. 